\title{
Detecting False Messages in the Smartphone Fault Reporting System
}

International Conference of Reliable Information and Communication Technology

IRICT 2019: Emerging Trends in Intelligent Computing and Informatics pp 759-768 |

Cite as

- Sharmiladevi Rajoo (1)

- Pritheega Magalingam (1) Email author (mpritheega.kl@utm.my)

- Ganthan Narayana Samy (1)

- Nurazean Maarop (1)

- Norbik Bashah Idris (2)

- Bharanidharan Shanmugam (3)

- Sundaresan Perumal (4)

1. Razak Faculty of Technology and Informatics, Universiti Teknologi Malaysia, , Skudai, Malaysia

2. International Islamic Universiti Malaysia, , Gombak, Malaysia

3. School of Engineering and Information Technology, Charles Darwin University, , Casuarina, Australia

4. Faculty of Science and Technology, Universiti Sains Islam Malaysia, , Gombak, Malaysia

Conference paper

First Online: 02 November 2019

- 263 Downloads

Part of the Advances in Intelligent Systems and Computing book series (AISC, volume 1073)

\section{Abstract}

The emergence of the Internet of Things (IoT) in Smart City allows mobile application developers to develop reporting services with an aim for local citizens to interact with municipalities regarding city issues in an efficient manner. However, the credibility of the messages sent rise as a great challenge when users intentionally send false reports through the application. In this research, an evidence detection framework is developed and divided into three parts that are a data source, IoT device's false text classification engine and output. Text-oriented digital evidence from an IoT mobile reporting service is analyzed to identify suitable text classifier and to build this framework. The Agile model that consists of define, design, build and test is used for the development of the false text classification engine. Focus given on text-based data that does not include encrypted messages. Our proposed framework able to achieve $97 \%$ of accuracy and showed the 
highest detection rate using SVM compared to other classifiers. The result shows that the proposed framework is able to aid digital forensic evidence experts in their initial investigation on detecting false report of a mobile reporting service application in the IoT environment.

\section{Keywords}

Internet of Things Smartphone Application Reporting services Smart City

Text classifiers

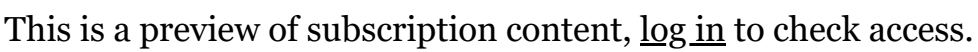

\section{Notes}

\section{Acknowledgement}

This work is supported in part by Redtone IOT Sdn. Bhd., Malaysia. We thank Redtone IoT staff members who provided insight and data that greatly assisted the research.

\section{References}

1. Walravens, N.: Mobile city applications for Brussels citizens: Smart City trends, challenges and a reality check. Telematics Inform. 32(2), 282-299 (2015)

CrossRef (https://doi.org/10.1016/j.tele.2014.09.004) Google Scholar (http://scholar.google.com/scholar_lookup? title=Mobile\%20city\%20applications\%2ofor\%20Brussels\%20citizens\%3A\%20Sm art\%20City\%2otrends\%2C\%20challenges\%20and\%20a\%2oreality\%20check\&aut hor=N.\%20Walravens\&journal=Telematics\%2oInform.\&volume=32\&issue=2\&pa ges $=282-299 \&$ publication_year $=2015)$

2. Cook, J.: Tell the City of Seattle about potholes and graffiti with new 'Find It, Fix It' app (2013). https://www.geekwire.com/2013/city-seattle-potholes-graffitifind-fix-it-app/ (https://www.geekwire.com/2013/city-seattle-potholes-graffitifind-fix-it-app/). Accessed 15 July 2019

3. (Greece), T.I.T.I.-M.G. Improve My City. http://smartcityapps.urenio.org/improve-my-city_en.html (http://smartcityapps.urenio.org/improve-my-city_en.html). Accessed 15 July 2019

4. REDtoneIOTSdn.Bhd., CitiAct - Your Key in Building the Next Smart City Applications (2016)

Google Scholar (https://scholar.google.com/scholar? q=REDtoneIOTSdn.Bhd.\%2C\%20CitiAct\%20\%E2\%80\%93\%20Your\%20Key\%20i n\%20Building\%20the\%20Next\%20Smart\%20City\%20Applications\%20\%282016 \%29) 
5. Aggarwal, C.C., Abdelzaher, T.: Social sensing. In: Managing and Mining Sensor Data, pp. 237-297. Springer, Heidelberg (2013)

Google Scholar (https://scholar.google.com/scholar?

q=Aggarwal\%2C\%20C.C.\%2C\%2oAbdelzaher\%2C\%20T.\%3A\%2oSocial\%2osensi ng.\%20In\%3A\%20Managing\%20and\%2OMining\%2oSensor\%2oData\%2C\%2opp. \%20237\%E2\%80\%93297.\%20Springer\%2C\%20Heidelberg\%20\%282013\%29)

6. Kantarci, B., Mouftah, H.T.: Trustworthy sensing for public safety in cloud-centric internet of things. IEEE Internet Things J. 1(4), 360-368 (2014)

CrossRef (https://doi.org/10.1109/JIOT.2014.2337886)

Google Scholar (http://scholar.google.com/scholar_lookup?

title=Trustworthy\%20sensing\%2ofor\%2opublic\%20safety\%2oin\%2ocloudcentric\%2ointernet\%20of\%2othings\&author=B.\%20Kantarci\&author=HT.\%20M ouftah\&journal=IEEE\%20Internet\%20Things\%20J.\&volume $=1 \&$ issue $=4 \&$ pages $=$ 360-368\&publication_year=2014)

7. Wang, D., Huang, C.: Confidence-aware truth estimation in social sensing applications. In: 2015 12th Annual IEEE International Conference on Sensing, Communication, and Networking (SECON). IEEE (2015)

Google Scholar (https://scholar.google.com/scholar? q=Wang\%2C\%20D.\%2C\%20Huang\%2C\%20C.\%3A\%2oConfidenceaware\%20truth\%20estimation\%20in\%20social\%20sensing\%20applications.\%20I n\%3A\%202015\%2012th\%20Annual\%20IEEE\%2OInternational\%2oConference\% 200n\%20Sensing\%2C\%20Communication\%2C\%20and\%20Networking\%20\%28S ECON\%29.\%20IEEE\%20\%282015\%29)

8. Marshall, J., Wang, D.: Towards emotional-aware truth discovery in social sensing applications. In: 2016 IEEE International Conference on Smart Computing (SMARTCOMP). IEEE (2016)

Google Scholar (https://scholar.google.com/scholar? q=Marshall\%2C\%20J.\%2C\%20Wang\%2C\%20D.\%3A\%20Towards\%2oemotionalaware\%20truth\%20discovery\%20in\%20social\%20sensing\%20applications.\%20In \%3A\%202016\%20IEEE\%2OInternational\%20Conference\%20on\%20Smart\%20Co mputing\%20\%28SMARTCOMP\%29.\%20IEEE\%20\%282016\%29)

9. Marshall, J., Syed, M., Wang, D.: Hardness-aware truth discovery in social sensing applications. In: 2016 International Conference on Distributed Computing in Sensor Systems (DCOSS). IEEE (2016)

Google Scholar (https://scholar.google.com/scholar?

q=Marshall\%2C\%2OJ.\%2C\%20Syed\%2C\%20M.\%2C\%2oWang\%2C\%2oD.\%3A\%2 oHardness-

aware\%2otruth\%20discovery\%20in\%20social\%20sensing\%20applications.\%20In \%3A\%202016\%20International\%2oConference\%20on\%2oDistributed\%20Comp uting\%20in\%2oSensor\%20Systems\%20\%28DCOSS\%29.\%20IEEE\%20\%282016 \%29)

10. Ghosh, N., et al.: A probabilistic approach for filtering out spam reports in a vehicular participatory sensing system. In: 2016 8th International Conference on Communication Systems and Networks (COMSNETS). IEEE (2016)

Google Scholar (https://scholar.google.com/scholar? q=Ghosh\%2C\%20N.\%2C\%20et\%20al.\%3A\%20A\%2oprobabilistic\%20approach\% 2ofor\%2ofiltering\%20out\%20spam\%2oreports\%20in\%20a\%2ovehicular\%2opart icipatory\%20sensing\%20system.\%20In\%3A\%202016\%208th\%20International\% 
20Conference\%20on\%20Communication\%20Systems\%20and\%20Networks\%20 \%28COMSNETS\%29.\%20IEEE\%20\%282016\%29)

11. Barnwal, R.P., et al.: Enhancing reliability of vehicular participatory sensing network: a bayesian approach. In: 2016 IEEE International Conference on Smart Computing (SMARTCOMP). IEEE (2016)

Google Scholar (https://scholar.google.com/scholar?

q=Barnwal\%2C\%2oR.P.\%2C\%20et\%20al.\%3A\%2oEnhancing\%2oreliability\%20o f\%20vehicular\%2oparticipatory\%2osensing\%2onetwork\%3A\%20a\%2obayesian\% 20approach.\%20In\%3A\%202016\%20IEEE\%2OInternational\%20Conference\%20 on\%20Smart\%20Computing\%20\%28SMARTCOMP\%29.\%20IEEE\%20\%282016 \%29)

12. Prandi, C., et al.: A trustworthiness model for crowdsourced and crowdsensed data. In: 2015 IEEE Trustcom/BigDataSE/ISPA. IEEE (2015)

Google Scholar (https://scholar.google.com/scholar? q=Prandi\%2C\%20C.\%2C\%20et\%20al.\%3A\%20A\%20trustworthiness\%2omodel\% 2ofor\%20crowdsourced\%20and\%20crowdsensed\%20data.\%20In\%3A\%202015\% 20IEEE\%20Trustcom\%2FBigDataSE\%2FISPA.\%20IEEE\%20\%282015\%29)

13. Pandey, U., Chakravarty, S.: A survey on text classification techniques for e-mail filtering. In: 2010 Second International Conference on Machine Learning and Computing (ICMLC). IEEE (2010)

Google Scholar (https://scholar.google.com/scholar?

q=Pandey\%2C\%20U.\%2C\%20Chakravarty\%2C\%20S.\%3A\%20A\%20survey\%200 n\%20text\%20classification\%20techniques\%2ofor\%20e-

mail\%2ofiltering.\%20In\%3A\%202010\%20Second\%20International\%2oConferen ce\%20on\%20Machine\%20Learning\%20and\%20Computing\%20\%28ICMLC\%29. \%20IEEE\%20\%282010\%29)

14. Tayal, D.K., et al.: Crime detection and criminal identification in India using data mining techniques. AI Soc. 3o(1), 117-127 (2015)

MathSciNet (http://www.ams.org/mathscinet-getitem?mr=3762708)

CrossRef (https://doi.org/10.1007/s00146-014-0539-6)

Google Scholar (http://scholar.google.com/scholar_lookup? title=Crime\%20detection\%20and\%20criminal\%2oidentification\%2oin\%20India \%2ousing\%20data\%20mining\%20techniques\&author=DK.\%20Tayal\&journal=AI \%20Soc. $\&$ volume $=30 \&$ issue $=1 \&$ pages $=117-127 \&$ publication_year $=2015$ )

15. Firoozjaei, M.D., J. Park, and H. Kim. Detecting false emergency requests using callers' reporting behaviors and locations. In: 2016 3oth International Conference on Advanced Information Networking and Applications Workshops (WAINA). IEEE (2016)

Google Scholar (https://scholar.google.com/scholar? q=Firoozjaei\%2C\%2OM.D.\%2C\%2OJ.\%2OPark\%2C\%2oand\%2OH.\%2OKim.\%20D etecting\%2ofalse\%2oemergency\%2orequests\%2ousing\%20callers\%E2\%80\%99\% 2oreporting\%2obehaviors\%20and\%2olocations.\%2oIn\%3A\%202016\%2030th\%2 oInternational\%20Conference\%20on\%20Advanced\%20Information\%20Network ing\%20and\%20Applications\%20Workshops\%20\%28WAINA\%29.\%20IEEE\%20\% 282016\%29)

16. Bhatti, F., et al.: A novel internet of things-enabled accident detection and reporting system for smart city environments. Sensors 19(9), 2071 (2019) CrossRef (https://doi.org/10.3390/s19092071) 
Google Scholar (http://scholar.google.com/scholar_lookup?

title=A\%2onovel\%2ointernet\%20of\%2othings-

enabled\%20accident\%2odetection\%20and\%2oreporting\%20system\%2ofor\%20s

mart\%20city\%20environments\&author=F.\%20Bhatti\&journal=Sensors\&volume=

19\&issue=9\&pages $=2071 \&$ publication_year $=2019$ )

17. Al-Zaidy, R., et al.: Mining criminal networks from unstructured text documents.

Digit. Invest. 8(3-4), 147-160 (2012)

CrossRef (https://doi.org/10.1016/j.diin.2011.12.001)

Google Scholar (http://scholar.google.com/scholar_lookup?

title=Mining\%20criminal\%2onetworks\%20from\%2ounstructured\%2otext\%2odo cuments\&author $=$ R.\%2OAl-

Zaidy\&journal=Digit.\%2OInvest.\&volume=8\&issue=3\%E2\%80\%934\&pages $=147-$

160\&publication_year=2012)

18. Marturana, F., et al.: A quantitative approach to triaging in mobile forensics. In:

2011 IEEE 1oth International Conference on Trust, Security and Privacy in

Computing and Communications (TrustCom). IEEE (2011)

Google Scholar (https://scholar.google.com/scholar?

q=Marturana\%2C\%20F.\%2C\%20et\%20al.\%3A\%20A\%20quantitative\%20approac

h\%20to\%2otriaging\%20in\%2Omobile\%2oforensics.\%20In\%3A\%202011\%20IEEE

\%2010th\%20International\%20Conference\%20on\%20Trust\%2C\%2oSecurity\%20

and\%20Privacy\%20in\%20Computing\%20and\%20Communications\%20\%28Trust

Com\%29.\%20IEEE\%20\%282011\%29)

19. Lakshmi, R.D., Radha, N.: Spam classification using supervised learning techniques. In: Proceedings of the 1st Amrita ACM-W Celebration on Women in Computing in India. ACM (2010)

Google Scholar (https://scholar.google.com/scholar?

q=Lakshmi\%2C\%20R.D.\%2C\%2ORadha\%2C\%20N.\%3A\%20Spam\%2oclassificati on\%20using\%20supervised\%2olearning\%2otechniques.\%2oIn\%3A\%20Proceedi

ngs\%20of\%20the\%201st\%20Amrita\%20ACM-

W\%20Celebration\%20on\%2oWomen\%20in\%20Computing\%2oin\%2oIndia.\%20 ACM\%20\%282010\%29)

20. Trivedi, S.K., Dey, S.: Interaction between feature subset selection techniques and machine learning classifiers for detecting unsolicited emails. ACM SIGAPP Appl.

Comput. Rev. 14(1), 53-61 (2014)

CrossRef (https://doi.org/10.1145/2600617.2600622)

Google Scholar (http://scholar.google.com/scholar_lookup?

title=Interaction\%2obetween\%2ofeature\%20subset\%2oselection\%2otechniques \%20and\%2omachine\%2olearning\%2oclassifiers\%2ofor\%2odetecting\%2ounsolici ted\%20emails\&author=SK.\%20Trivedi\&author=S.\%20Dey\&journal=ACM\%2OSI GAPP\%2OAppl.\%20Comput.\%20Rev.\&volume=14\&issue=1\&pages=53-

61\&publication_year=2014)

21. Wang, S., et al.: Detecting android malware leveraging text semantics of network flows. IEEE Trans. Inf. Forensics Secur. 13(5), 1096-1109 (2018)

CrossRef (https://doi.org/10.1109/TIFS.2017.2771228)

Google Scholar (http://scholar.google.com/scholar_lookup?

title=Detecting\%20android\%20malware\%2oleveraging\%2otext\%20semantics\%2 oof\%2onetwork\%20flows\&author=S.\%20Wang\&journal=IEEE\%2OTrans.\%20Inf 
\%20Forensics\%20Secur.\&volume=13\&issue $=5$ \&pages=1096-

1109\&publication_year=2018)

22. Gautam, G., Yadav, D.: Sentiment analysis of twitter data using machine learning approaches and semantic analysis. In: 2014 Seventh International Conference on Contemporary computing (IC3). IEEE (2014)

Google Scholar (https://scholar.google.com/scholar?

q=Gautam\%2C\%2oG.\%2C\%2oYadav\%2C\%2oD.\%3A\%2oSentiment\%20analysis

\%20of\%2otwitter\%20data\%2ousing\%2omachine\%2olearning\%20approaches\%2

oand\%20semantic\%20analysis.\%20In\%3A\%202014\%20Seventh\%20Internationa 1\%20Conference\%20on\%20Contemporary\%20computing\%20\%28IC3\%29.\%20IE EE\%20\%282014\%29)

23. Munassar, N.M.A., Govardhan, A.: A comparison between five models of software engineering. IJCSI 5, 95-101 (2010)

Google Scholar (http://scholar.google.com/scholar_lookup?

title=A\%20comparison\%2obetween\%2ofive\%2omodels\%20of\%2osoftware\%20e ngineering\&author=NMA.\%20Munassar\&author=A.\%20Govardhan\&journal=IJC SI\&volume $=5 \&$ pages $=95-101 \&$ publication $\_$year $=2010$ )

24. Torgo, L.: Data mining with R. Learning by case studies. University of Porto, LIACC-FEP (2003). http://www.dcc.fc.up.pt/ ltorgo/DataMiningWithR/ (http://www.dcc.fc.up.pt/\%7eltorgo/DataMiningWithR/). Accessed 1 July 2019

25. Panigrahi, P.K.: A comparative study of supervised machine learning techniques for spam e-mail filtering. In: 2012 Fourth International Conference on Computational Intelligence and Communication Networks. IEEE (2012)

Google Scholar (https://scholar.google.com/scholar? q=Panigrahi\%2C\%2OP.K.\%3A\%20A\%20comparative\%2ostudy\%20of\%20supervi sed\%2omachine\%2olearning\%2otechniques\%2ofor\%2ospam\%2oemail\%2ofiltering.\%20In\%3A\%202012\%20Fourth\%2oInternational\%20Conferenc e\%20on\%20Computational\%2oIntelligence\%20and\%20Communication\%20Net works.\%20IEEE\%20\%282012\%29)

26. Brindha, S., Prabha, K., Sukumaran, S.: A survey on classification techniques for text mining. In: 2016 3rd International Conference on Advanced Computing and Communication Systems (ICACCS). IEEE (2016)

Google Scholar (https://scholar.google.com/scholar? q=Brindha\%2C\%20S.\%2C\%2OPrabha\%2C\%20K.\%2C\%20Sukumaran\%2C\%2oS. \%3A\%20A\%20survey\%20on\%2oclassification\%2otechniques\%2ofor\%2otext\%20 mining.\%20In\%3A\%202016\%203rd\%20International\%20Conference\%20on\%20 Advanced\%20Computing\%20and\%20Communication\%20Systems\%20\%28ICAC CS\%29.\%20IEEE\%20\%282016\%29)

27. Rajoo, S., et al.: A comparative study of text classifier for mobile crowdsensing applications. Adv. Sci. Lett. 24(1), 686-689 (2018)

CrossRef (https://doi.org/10.1166/asl.2018.11788)

Google Scholar (http://scholar.google.com/scholar_lookup?

title=A\%20comparative\%20study\%20of\%20text\%20classifier\%2ofor\%20mobile \%20crowdsensing\%20applications\&author=S.\%20Rajoo\&journal=Adv.\%20Sci.\% 20Lett.\&volume=24\&issue $=1 \&$ pages $=686-689 \&$ publication_year=2018)

28. Gopal, S., et al.: Statistical learning for file-type identification. In: 2011 10th International Conference on Machine Learning and Applications and Workshops 
(ICMLA). IEEE (2011)

Google Scholar (https://scholar.google.com/scholar?

q=Gopal\%2C\%20S.\%2C\%20et\%20al.\%3A\%2oStatistical\%2olearning\%2ofor\%2of

ile-

type\%20identification.\%20In\%3A\%202011\%2010th\%20International\%20Confere nce\%20on\%20Machine\%20Learning\%20and\%20Applications\%20and\%20Works

hops\%20\%28ICMLA\%29.\%20IEEE\%20\%282011\%29)

\section{Copyright information}

(C) Springer Nature Switzerland AG 2020

\section{About this paper}

Cite this paper as:

Rajoo S. et al. (2020) Detecting False Messages in the Smartphone Fault Reporting System. In: Saeed F., Mohammed F., Gazem N. (eds) Emerging Trends in Intelligent Computing and Informatics. IRICT 2019. Advances in Intelligent Systems and Computing, vol 1073. Springer, Cham

- First Online 02 November 2019

- DOI https://doi.org/10.1007/978-3-030-33582-3_71

- Publisher Name Springer, Cham

- Print ISBN 978-3-030-33581-6

- Online ISBN 978-3-030-33582-3

- eBook Packages Intelligent Technologies and Robotics Intelligent Technologies and Robotics $(\underline{\text { Ro }})$.

- Buy this book on publisher's site

- Reprints and Permissions

\section{Personalised recommendations}

\section{SPRINGER NATURE}

(C) 2020 Springer Nature Switzerland AG. Part of $\underline{\text { ppringer Nature. }}$

Not logged in Not affiliated 210.195.47.55 